\title{
Culture and Change Blindness
}

\author{
Takahiko Masuda ${ }^{\mathrm{a}}$, Richard E. Nisbett ${ }^{\mathrm{b}}$ \\ a Department of Psychology, University of Alberta \\ ${ }^{\mathrm{b}}$ Department of Psychology, University of Michigan
}

Received 11 November 2004; received in revised form 17 October 2005; accepted 8 November 2005

\begin{abstract}
Research on perception and cognition suggests that whereas East Asians view the world holistically, attending to the entire field and relations among objects, Westerners view the world analytically, focusing on the attributes of salient objects. These propositions were examined in the change-blindness paradigm. Research in that paradigm finds American participants to be more sensitive to changes in focal objects than to changes in the periphery or context. We anticipated that this would be less true for East Asians and that they would be more sensitive to context changes than would Americans. We presented participants with still photos and with animated vignettes having changes in focal object information and contextual information. Compared to Americans, East Asians were more sensitive to contextual changes than to focal object changes. These results suggest that there can be cultural variation in what may seem to be basic perceptual processes.
\end{abstract}

Keywords: Culture; Attention; Change blindness; Change detection; Holistic vs. Analytic thought; Japanese; Americans; Cultural psychology

\section{Introduction}

Westerners and East Asians differ in their judgments about causality for events, both physical and social. Westerners tend to locate causality in the object, whereas East Asians are more likely to call on the field or context as well (e.g., Morris \& Peng, 1994; Norenzayan \& Nisbett, 2000; Peng \& Knowles, 2003).

Nisbett and his colleagues (Ji, Peng, \& Nisbett, 2000; Nisbett, 2003; Nisbett, Peng, Choi, \& Norenzayan, 2001) have argued that these different tendencies in causal attribution, as well as other perceptual and cognitive differences between Asians and Westerners, are due in part to differences in attention to the object versus the context. East Asians live in highly interdependent societies. They must attend to their relationships with other people before they can take action with respect to personal goals. Westerners, in contrast, live in more independent societ-

Correspondence should be addressed to Takahiko Masuda, Department of Psychology, University of Alberta, P-355, Biological Sciences Building, Edmonton, AB, Canada, T6G 2E9. E-mail: tmasuda@ualberta.ca 
ies allowing relatively socially unimpeded attention to personal goals with respect to important objects. Because attention is directed toward the social world for East Asians, causality is seen to inhere substantially in the context. As Markus and Kitayama (1991, p. 246) put it, "If one perceives oneself as embedded within a larger context of which one is an interdependent part, it is likely that other objects or events will be perceived in a similar way." Because attention is focused on salient objects for Westerners, in contrast, it is natural for them to attribute causality to objects.

Masuda and Nisbett (2001) obtained evidence for the predicted attentional differences by showing American and Japanese college students animated vignettes of underwater scenes and subsequently asking the participants what they had seen. They found that the American participants tended to begin by referring to the most salient objects in the vignettes (that is, the largest, brightest, most rapidly moving objects). They were likely to say, "I saw what looked like a trout swimming off to the left." Japanese participants were much more likely to begin with the context. They were likely to say, "I saw what looked like a stream; the water was green; there were rocks on the bottom." The Japanese participants reported more than $60 \%$ more details about the context than did the American participants.

The Japanese participants also appeared to see the objects in relation to the context. After participants had been shown eight vignettes, they were shown individual objects. Half of these had been seen before and half had not. Some were shown with their original backgrounds and some were shown in novel backgrounds. Japanese participants showed a "binding" effect (Chalfonte \& Johnson, 1996); that is, the accuracy of their reports as to whether or not they had seen an object was thrown off if they saw the object in an environment different from the one in which it had initially appeared. American participants were not affected by the background manipulation.

It would be valuable to have additional evidence on the question of whether the attention of Westerners and East Asians is directed differently. One paradigm that seems a promising one for investigation is the so-called change-blindness paradigm. Previous research has shown that people often fail to recognize marked changes in their surroundings (Simons, 2000; Simons \& Levin, 1998). For example, when people are asked to watch a videotape and count the number of ball passes between players, they consistently fail to notice the insertion of an unusual character into the scene, such as a person carrying an umbrella or even a person wearing a gorilla costume (Simons \& Chabris, 1999). Change blindness is evident even when participants are explicitly asked to search for changes in the visual field. For example, various researchers have used the "flicker" paradigm in which an original image repeatedly alternates with a second image until the participant recognizes the change (Rensink, O'Regan, \& Clark, 1997). Participants often take a great deal of time to detect major changes in a given scene. A generalization emerging from this research is that people are likely to detect changes in salient, focal objects faster than in objects in the periphery or context (Rensink et al., 1997; Scholl, 2000). In this research, we compared the change blindness of Americans and Japanese. We expected to find the Japanese to be more focused on changes in the context than Americans.

If the anticipated differences were to be found, we believe that the explanation would be found in cultural differences between East Asians and Westerners. First, East Asians are socialized to attend to contexts, including both physical and socioemotional contexts. Bornstein, Toda, Azuma, Tamis-LeMonda, and Ogino (1990) and Fernald and Morikawa (1993) studied 
Japanese and American mothers and their infants and found that the American mothers directed their infants' attention primarily to objects, whereas Japanese mothers directed their infants' attention alternately to objects and to the mother's face, thereby encouraging attention to social factors at the same time the object was being examined. The Japanese mothers also emphasized interactive social routines ("I give it to you. You give it to me. Yes! Thank you"). Tardif, Shatz, and Naigles (1997) found that American mothers use more nouns (i.e., words referring to objects) when speaking with their infants. In contrast, Chinese mothers use relatively more verbs (i.e., words primarily referring to relationships in the environment or between the infant or mother and the environment). Second, as we will show later, there is evidence that Asian-built environments are more complex than Western environments. The complexity of the environment could serve to draw habitual attention to the context as opposed to salient objects (Chun, 2000). Either or both cultural factors could prompt differential attention of Asians and Westerners.

\section{Experiment 1}

\subsection{Method}

\subsubsection{Materials}

Following the original flicker paradigm (Rensink et al., 1997), we created a set of flicker sequences of images, in which an original image and a modified image were presented in the sequence $\mathrm{A}, \mathrm{A}^{\prime}, \mathrm{A}, \mathrm{A}^{\prime} \ldots$, with a blank field presented between two images (Fig. 1). Each image was presented for $560 \mathrm{msec}$, and the blank field was presented for $80 \mathrm{msec}$. Using PsyScope, 30 pairs of slightly different color images of realistic industrial scenes were prepared - an airport, a construction site, a town, a farm, and a harbor. Each image contained focal objects (e. g., foregrounded machines) as well as contexts (e.g., background buildings).

In each pair of images, there occurred only one change, which was either a change in focal object information (e.g., focal vehicle's color) or a change in contextual information (e. g., changes in the location of clouds). We believe that the objects that underwent changes were all typical of the scenes they had appeared in (see Appendix A). The alternation continued for a minute, and images disappeared if participants could not detect the changes within a minute.

\subsubsection{Participants and procedure}

Thirty American students (15 women and 15 men) and 36 East Asian international students (Chinese, Japanese, or Korean; 17 women and 19 men) at the University of Michigan participated in the experiment as a course requirement or for $\$ 10 .{ }^{1}$ About two thirds of participants in both populations were paid. After greeting the participants, the experimenter escorted each to a cubicle. A 13-in. laptop computer (Macintosh Powerbook G3) presented the images, which were 22 -cm wide and $16.5-\mathrm{cm}$ high. Viewing distance $(30 \mathrm{~cm})$ and angle (about $40.7^{\circ} \times 31.5^{\circ}$ ) from the monitor were standardized by asking participants to sit in chairs with their chins resting on a small platform. The experimenter explained that the participants' task was to view quick alternations of a pair of images and to identify the differences between the first and second images. As may be seen in Appendix A, participants were presented 30 different pairs of 
A

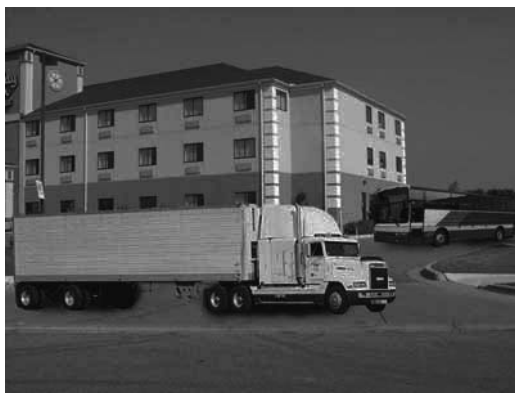

B

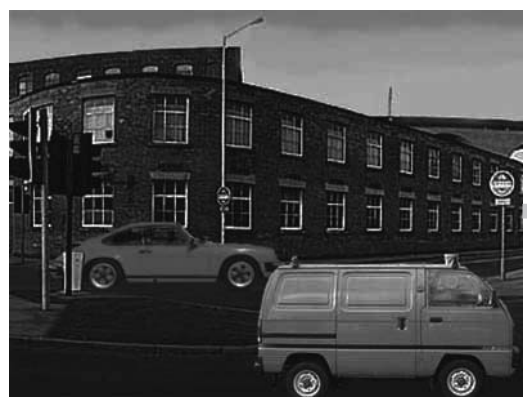

$A^{\prime}$

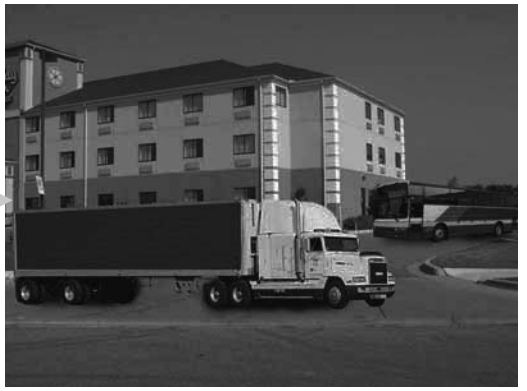

B'

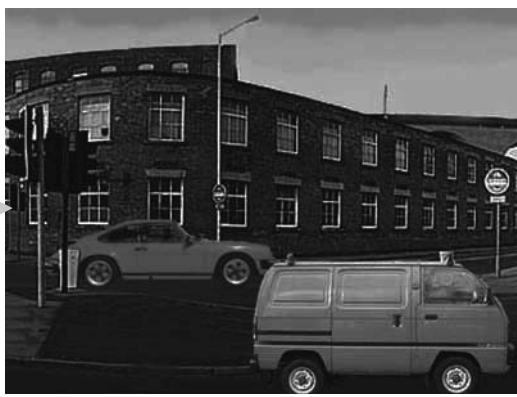

Fig. 1. Examples of changes in industrial scenes. The wheel of the bicycle in Picture A disappears in Picture A'. The location of sidewalk at the left bottom area in Picture B has been changed in Picture B'.

scenes. The instructions were identical for both cultural groups. (For this and the other two studies, we used the back translation technique to assure equivalence.) Participants were asked to press a key when they recognized the change and later to report it orally. Experimenters checked whether their identification of changes were correct or not. Only $4.5 \%$ of the responses were coded as a "miss" or "run out of time." 2 We analyzed only the data for which participants correctly detected the changes within $60 \mathrm{sec}$.

\subsection{Results}

\subsubsection{Manipulation check}

After the experiment, we asked the same participants to identify what they regarded as the central objects of the 30 scenes. The results of chi-square analyses indicated that, for all $30 \mathrm{im}-$ ages, there were no significant cultural differences in the objects that the participants identified as central. Overall, $84 \%$ of Americans and $83 \%$ of East Asians indicated that they identified as central objects those that corresponded to the experimenter's intention. ${ }^{3}$

\subsubsection{Change detection}

A 2 (culture: Americans vs. East Asians) $\times 2$ (type of change: object vs. context) analysis of variance (ANOVA) of reaction time for the identification of changes shown in Fig. 2 revealed that 


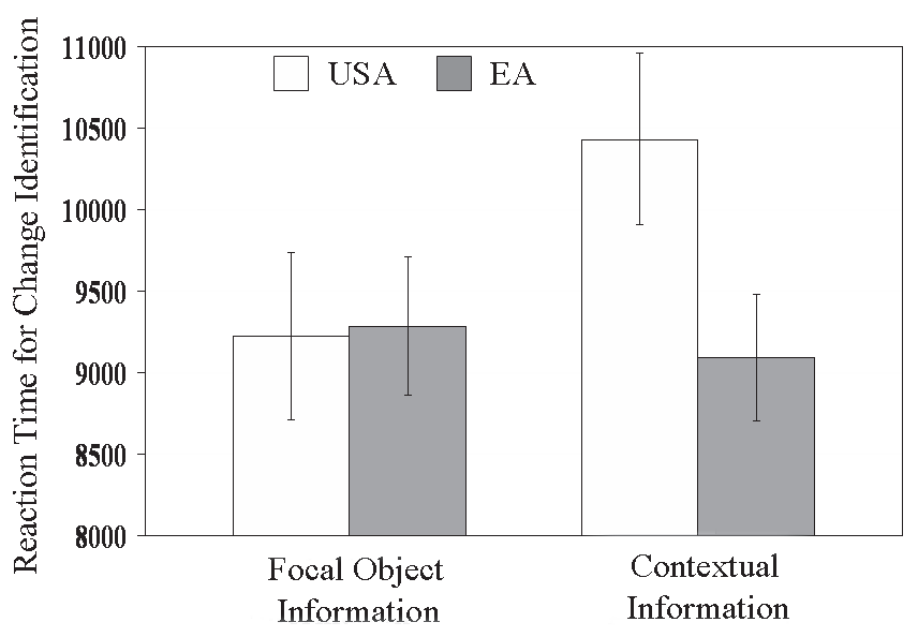

Fig. 2. Reaction time for change identification in Experiment 1.

there was an interaction of culture and type of change, $F(1,64)=3.96, p=.05 .{ }^{4} \mathrm{~A}$ planned contrast analysis indicated that East Asians detected changes in contextual information faster than did Americans, $F(1,64)=4.02, p<.05$. Another contrast showed that American participants detected changes in the focal objects faster than changes in contextual information, $F(1,64)=5.45$, $p<.03$. East Asian participants detected context changes as rapidly as object changes.

These results indicate that East Asian participants attended to the contextual information more than did Americans. As in previous research, American detection of object changes was more rapid than detection of context changes, but this was not true for East Asian participants. Thus the generalization in the change-blindness literature that focal object changes are more detectable than context changes held only for the American participants in this study.

\section{Experiment 2}

The data from the flicker paradigm provides us with evidence of cultural variation in ways of seeing visual scenes. However, the task dealt only with still images. Several researchers have investigated change blindness for continuous movement or dynamic stimuli in the real world (e.g., Levin \& Simons, 1997; Simons \& Chabris, 1999; Simons \& Levin, 1998). Using their basic methodology, we showed a movie clip for $20 \mathrm{sec}$. Instead of using the most common measure of change detection in previous studies, namely, reaction time, we examined the number of detected changes in two types of information-focal object information and contextual information. Because perception of naturalistic scenes was our primary interest, we developed animated vignettes in which realistic objects were shown against meaningful backgrounds. In these vignettes changes occurred simultaneously in various respects, including the attributes of focal objects as well as backgrounds and the location and movement of objects. We anticipated that cultural differences in the detection of change would be particularly likely to emerge under 
conditions of heavy attentional load, in which multiple changes occur in both the focal objects and the context of the scene (cf. Kelley, Chun, \& Chua, 2003; Ro, Russell, \& Lavie, 2001).

\subsection{Method}

\subsubsection{Materials}

We created five pairs of animated color vignettes, using Macromedia's Director 7 (Macromedia, San Francisco, California), which were slightly different from each other. Black-and-white stills from the airport vignettes are shown in Fig. 3. As may be seen in Appendix B, all animated vignettes included three to four focal objects (e.g., aircraft), some of which moved and the rest of which were stable and situated either in the foreground or in the middle-range area. In addition, all scenes included several background objects, such as ground, sky, and buildings. Again, the objects that underwent changes were all typical of the scenes they had appeared in. The length of each animation was $20 \mathrm{sec}$. Participants were asked to detect changes between the first and second animated sequence of the same scene.

A

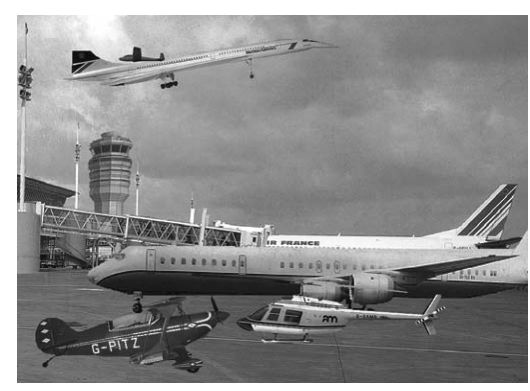

$\mathrm{C}$

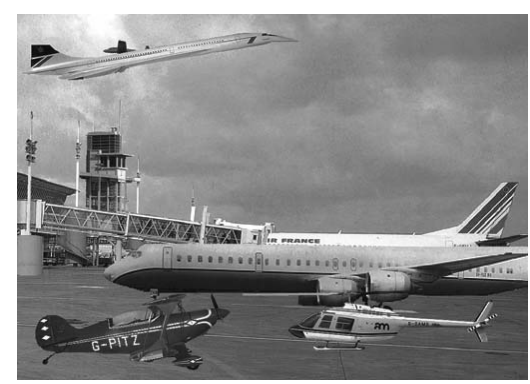

B

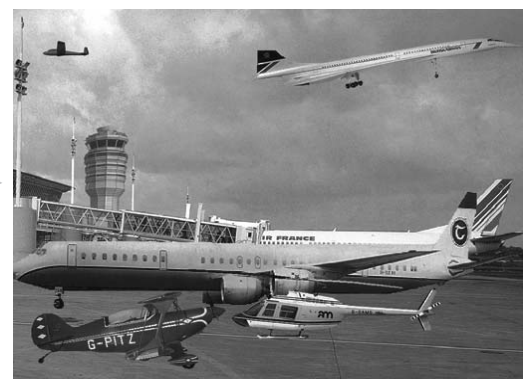

$\mathrm{D}$

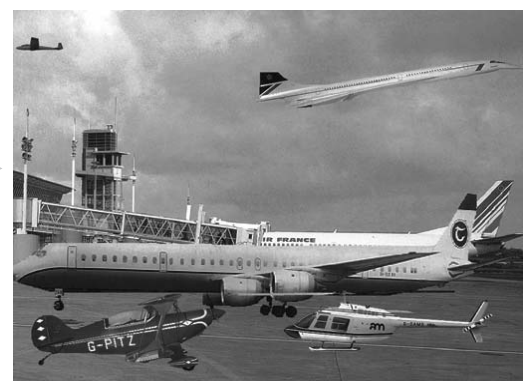

Fig. 3. An example of a vignette (a set of movie clips) used in Experiment 2, consisting of four distinct pictures. Picture A represents the first part of Airport clip 1. Picture B represents the last part of Airport clip 1. This sequence was presented for $20 \mathrm{sec}$. After the $2 \mathrm{sec}$ break, Airport clip 2 was presented. Picture $\mathrm{C}$ represents the first part of Airport clip 2, and Picture D represents the last part of Airport clip 2. Between Clip 1 and Clip 2, various changes in a foreground object's attributes, in a foreground object's location, in background, and in a foreground object's movement occur. 
A. Changes in focal object information: reference to the changes of either moving or stable machines' properties such as shape, color. and number.

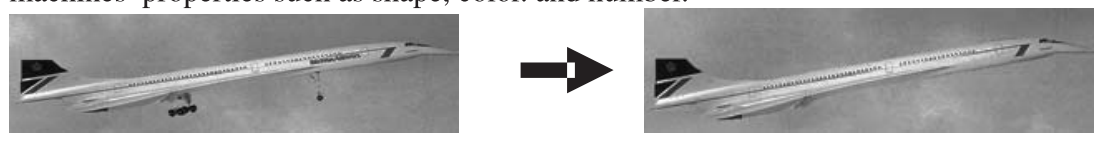

B. Changes in contextual/relational information: reference to changes in background, in objects' location, and in object's movement.

(a) Changes in background: reference to the changes of background buildings, grounds, clouds, and mountains.
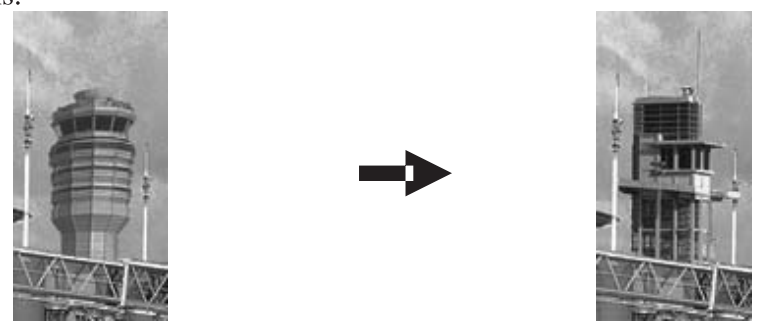

(b) changes in objects' location: reference to the changes of objects' position on the screen.
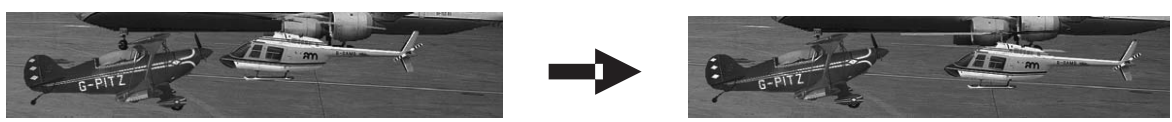

(c) Changes in objects' movement: reference to the changes of the machines' moving areas.

Fig. 4. Examples of changes in focal object information and in contextual information. All pictorial information is presented in Fig. 3. In section A "changes in focal objects information," the airline logo and the landing gear depicted in the left picture have been removed in the right picture. In section B "changes in contextual information," there are three subcategories. In (a) "changes in background," the helicopter in the left picture was replaced toward the right of its position in the right picture. In (b) "changes in objects' location," the control tower situated in the background appears as a round building (left), having appeared as a rectangular building (right). The last line indicates the definition of (c) "changes in objects' movement."

Fig. 4 illustrates the two types of change manipulated: change in focal object information (e.g., machines) and change in contextual information (e.g., background objects and object location).

The same 17-in. (about $43 \mathrm{~cm}$ ) Macintosh color monitor (about $33 \mathrm{~cm}$ wide $\times 25 \mathrm{~cm}$ high) and computer (Macintosh G3) were used in both the American and Japanese laboratories. Viewing distance $\left(40 \mathrm{~cm}\right.$ ) and angle (about $44.8^{\prime} \times 34.7$ ) from the monitor was standardized by asking participants to sit in chairs with their chins resting on a small platform.

\subsubsection{Participants and procedure}

The groups were matched as closely as possible across the two countries. Nineteen undergraduate American students at the University of Michigan ( 9 women and 10 men, age range: 
18-22, $M=19.4$ ) and 18 undergraduate Japanese students at Kyoto University in Japan (8 women and 10 men, age range: $18-21, M=19.3$ ) participated in the experiment as a course requirement. After greeting participants, the experimenter escorted each one to a cubicle. The experimenter explained that the participant's task was to view five vignette pairs and to determine the differences between the first and second vignettes. Participants were allowed to watch each pair of vignettes four times. After each viewing of a pair of sequences, participants were asked to describe the changes they saw on a sheet of paper in as specific and concrete terms as possible. The participants were also allowed to correct their answers if they thought they had made a mistake. Half of the participants from each culture watched the animations in the order listed previously and half in the reverse order.

\subsubsection{Data coding}

Three Japanese-English bilingual coders (two Japanese-born, one American-born) coded each sentence as referring to one of the two categories of change. One coded the entire data set, whereas the two others coded half of the data. Agreement was 94\%. To ensure that language did not influence their coding, a bilingual translator translated one third of the Japanese data into English and one third of the American data into Japanese. A monolingual American and a monolingual Japanese then coded these data. Their agreement was 93\%. We used the first set of coded data for the analyses (with disagreements among coders decided by the first author). Coders agreed that $5.4 \%$ of the responses were incorrect answers. ${ }^{5}$ We examined only the correct responses in further analyses.

\subsection{Results}

For each participant, we computed the mean number of detections of focal object changes and the mean number of detections of context changes across all five scenes and summarized across individual means to determine the group tendency in attention allocation. The mean number of changes detected in focal object information and contextual information is shown in Fig. 5. A 2 (culture) $\times 2$ (object vs. context) ANOVA showed that there was an interaction between culture and allocation of attention, $F(1,35)=9.44, p<.005$. Simple effect analyses revealed that Japanese participants were more likely than American participants to detect changes in contextual information, $F(1,35)=5.68, p<.03$. American participants were marginally more likely than Japanese participants to detect changes in focal object information, $F(1,35)=2.92, .05<p<.10$.

\section{Experiment 3}

In Experiment 3, we attempted to replicate and extend Experiment 2. Except for the airport and construction scenes, the stimuli used in Experiment 2 consisted of distinctly American scenes. ${ }^{6}$ In Experiment 3, we added additional scenes consisting of realistic pictorial materials that appeared to be Japanese. The two types of material differed in many ways, but the most relevant for present purposes was that the American scenes were object oriented. This means 


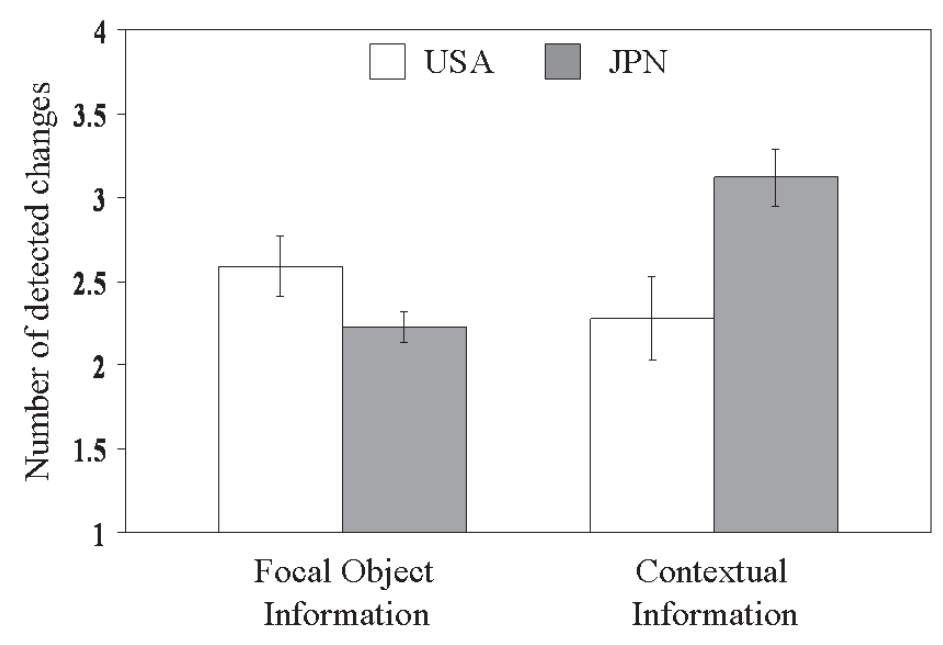

Fig. 5. Average number of detected changes for all vignettes in Experiment 2.

that there were relatively few objects, the objects were highly distinct and salient, and the backgrounds were relatively simple. The Japanese scenes, in contrast, were highly complex, containing many objects that were relatively difficult to distinguish from their backgrounds. These differences capture what the work of Miyamoto, Nisbett, and Masuda (in press) has shown to be ecologically typical of the respective countries. These investigators photographed 1,000 scenes close to randomly chosen schools, hotels, and post offices in large cities, medium-size cities, and villages in the United States and Japan. In all town sizes they found the Japanese environments to consist of more objects and to be more complex, as measured both subjectively and objectively.

\subsection{Method}

\subsubsection{Materials and preliminary experiment}

In addition to previous vignettes created using basically American pictorial sources, we added two Japanese scenes: a Japanese town and a Japanese farm (see Appendix B). To determine the cultural specificity of each vignette, we asked 31 American participants and 31 Japanese participants to evaluate whether these pictures represented Japanese scenery, American scenery, or scenery that could be seen in both cultures. To make their evaluations, participants used a 7-point scale $(1=$ strongly represents Japanese scene, $7=$ strongly represents American scene). As we anticipated, the airport scene and the construction scenes were evaluated as common to both cultures (average $M=4.32$ ). The scenes of a town, a farm, and a harbor from Experiment 2 were regarded as more likely to represent American scenery (average $M=5.13$ ). Finally, the new Japanese town scene and the new Japanese farm scene were regarded as more likely to represent Japanese scenery (average $M=1.73$; see Fig. 6). 
A

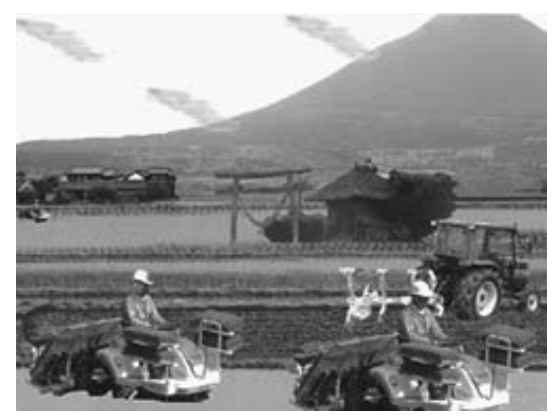

$\mathrm{C}$

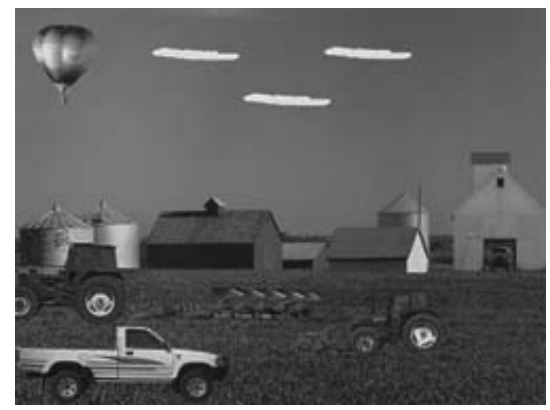

B

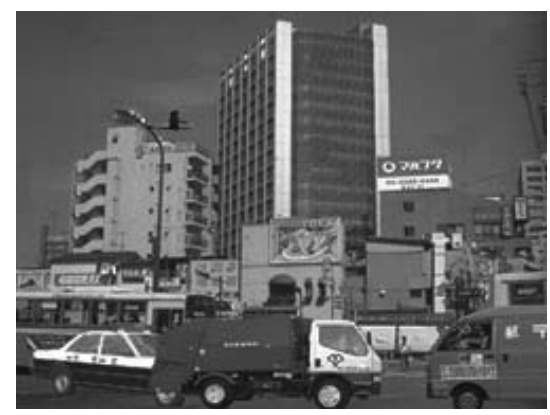

$\mathrm{D}$

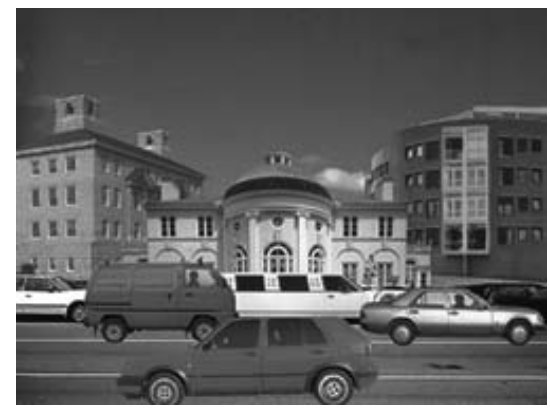

Fig. 6. Example images used in Experiment 2. Images A (farm) and B (town) represent Japanese scenes. Images C (farm) and D (town) represent American scenes. Original stimuli are animated vignettes.

\subsubsection{Participants and procedure}

Again, the groups were matched as closely as possible across the two countries. Twenty-eight undergraduate American students at the University of Michigan (15 women and 13 men, age range: $18-21, M=18.8$ ) and 32 undergraduate Japanese students at Kyoto University in Japan (17 women and 15 men, age range: $18-25, M=19.9)$ participated in the experiment as a course requirement. As in Experiment 2, participants saw five vignettes. All the participants saw the two culturally neutral scenes-the airport and the construction site vignettes. In addition, participants saw three of the culturally specific scenes-either two American and one Japanese or two Japanese and one American.

\subsubsection{Data coding}

The same coding procedures used for Experiment 2 were applied. Agreement among three coders was $93 \%$. Agreement of a monolingual American and a monolingual Japanese was $92 \%$. Coders agreed that $5.3 \%$ of the responses were incorrect answers. We analyzed only the correct responses. 


\subsection{Results}

The number of detected changes in both categories is shown in Fig. 7. A 2 (culture) $\times 2$ (object vs. context) ANOVA for the average reports for the five scenes showed that there was a significant interaction between culture and allocation of attention, $F(1,58)=22.72, p$ $<.001$. Simple analyses revealed that Japanese participants were more likely to detect changes in contextual information than were Americans $F(1,58)=16.17, p<.001$. Americans were more likely to detect changes in focal object information than the Japanese, $F(1$, $58)=5.63, p<.02$. The patterns for culturally neutral scenes and culturally specific scenes were similar, with the exception that the difference in change detection for focal objects for culturally neutral scenes was slight and insignificant. Overall, we replicated the findings of Experiment 2 but with much stronger results-undoubtedly primarily due to the increased number of participants.

We also found a significant interaction between allocation of attention and type of cultural scene, $F(1,58)=128.62, p<.001{ }^{7}$ Fig. 8 shows the responses of both Americans and Japanese to U.S. scenes and the responses of both groups to Japanese scenes. Simple effect analyses revealed that both Japanese and American participants detected changes in focal object information in American scenes more often than in Japanese scenes, $F(1,58)=11.96, p<.001$. Moreover, both Japanese and American participants detected changes in contextual information in Japanese scenes more often than in American scenes, $F(1,58)=7.74, p<.001$. There was no significant interaction between culture and type of scene. Thus, American scenes appear to have facilitated attention to foreground objects, whereas Japanese scenes facilitated attention to relationships and background. These results suggest that environments characteristic of different cultures direct people's attention differently.

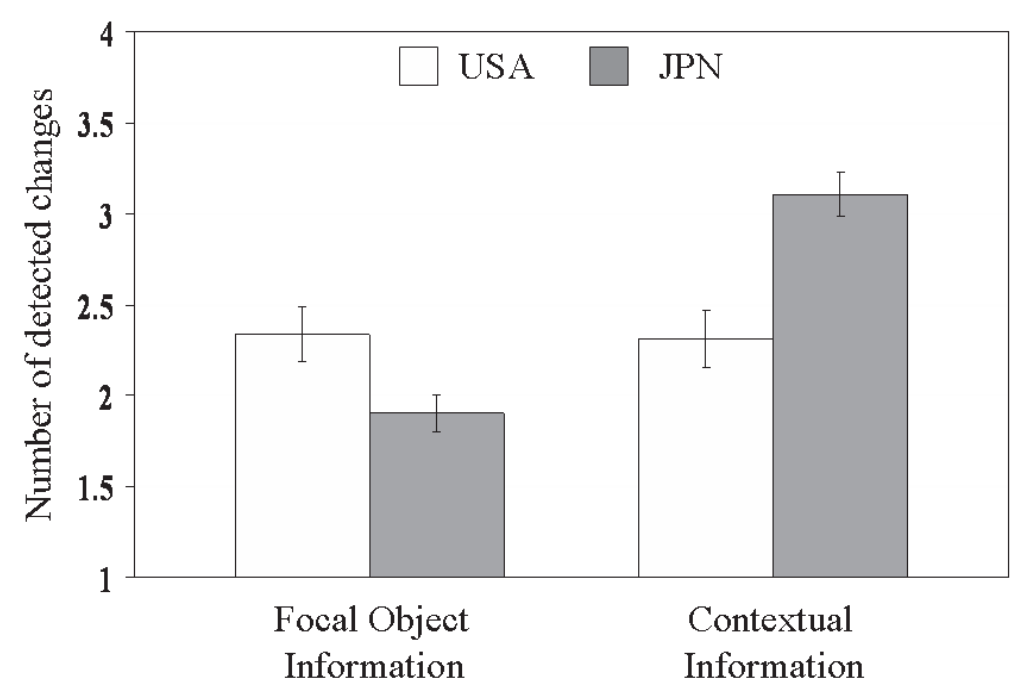

Fig. 7. Average number of detected changes for all vignettes in Experiment 3. 


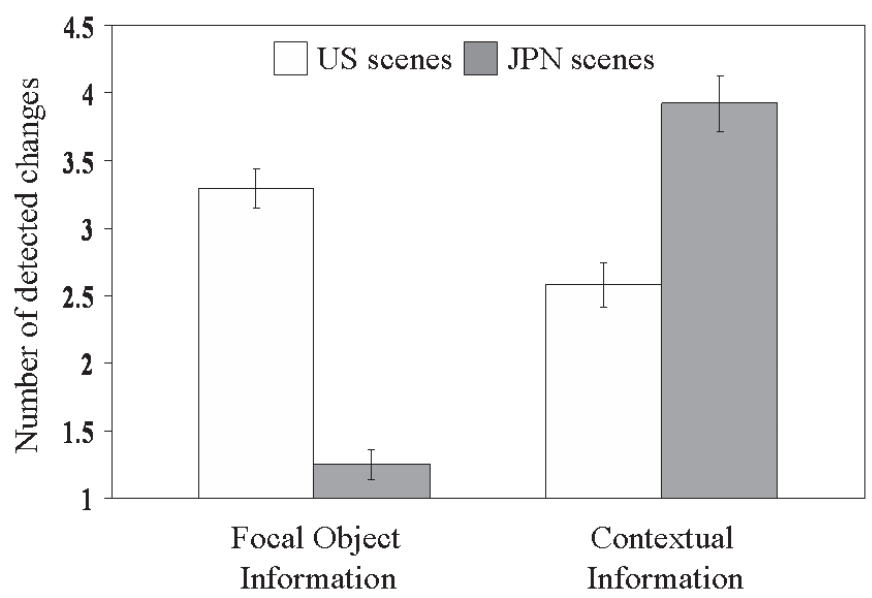

Fig. 8. Average number of detected changes for culturally specific vignettes in Experiment 3.

\section{General discussion}

This research provides evidence that cultural variations are observable even in patterns of attention that one might assume to be governed by basic, invariant psychological processes. In Experiment 1 we found that Japanese participants detected changes in context information more rapidly than did American participants, whereas there was no difference in how rapidly the two groups detected changes in object information. As has been found by the research of others, Americans detected object changes more rapidly than changes in context information, but the two types of information were detected equally rapidly by Japanese participants. In Experiments 2 and 3 we found that Americans detected more object changes than Japanese but that Japanese detected far more context changes than object changes.

\section{Reasoning style, visual experience, and attention}

Why might Easterners and Westerners allocate their attention differently? Past theorizing by Nisbett and his colleagues (Nisbett, 2003; Nisbett, Peng, Choi, \& Norenzayan, 2001) has emphasized socialization differences that begin very early. Although it is likely that such socialization differences are indeed important, these results suggest that the affordances of the environments that Asians and Westerners confront also play a role. Miyamoto et al. (in press) sampled 1,000 scenes from American and Japanese towns and found Japanese towns to contain more objects and be more complex. Eastern environments thus may focus attention on context. That this hypothesis is plausible is indicated by the results of a study by Miyamoto et al. They primed participants either with photographs they had taken of American scenes or with photographs of their Japanese scenes. They then examined American and Japanese re- 
sponses to a change-blindness task using the culturally neutral pictures from Experiment 3 of this research. Participants—both American and Japanese-primed with Japanese scenes saw more changes in contexts than did participants primed with American scenes. This result is consistent with findings indicating that learned visual experience pertaining to contextual factors shapes the way individuals allocate attention (Chun, 2000).

Although there is evidence that environmental affordances make a contribution to the cultural differences in attention that we have found, we do not believe this is the whole story. There are many studies showing greater attention to context by Asians, not only in other attention domains (Ishii, Reyes, \& Kitayama, 2003), but also in memory (Hedden et al., 2000; Liu \& Nisbett, 2005; Masuda \& Nisbett, 2001) and even inference processes, including causal reasoning (Choi, Nisbett, \& Norenzayan, 1999; Masuda \& Kitayama, 2004; Miyamoto \& Kitayama, 2002; Morris \& Peng, 1994; Norenzayan \& Nisbett, 2000). Thus, although it is likely that ecological factors play a role in attentional differences, the fact that parents socialize different patterns of attention and the fact that cultural differences are found for a wide variety of attention and reasoning tasks indicate that environmental affordances should not be regarded as the sole cause of the effects we have reported.

\section{Cultural difference in attention}

Are the cultural variations found in these studies attributable to genuine differences in attentional processes? We believe there is good evidence for that. The responses in Experiments 2 and 3 were verbal descriptions for which it may not be possible to completely eliminate differences in language or conversational rules. But responses in Experiment 1 were reaction times. Moreover, the findings of eye-tracking studies give credence to the assertion that there are genuine attentional differences. For example, Masuda et al. (2005) presented participants with a series of cartoon images, consisting of a target figure in the center and four background figures in the peripheral area, and asked participants to judge the central figure's emotion based on his facial expression. The results indicated that East Asians were more likely than their North American counterparts to allocate their attention to the peripheral figures' facial expressions and that their judgment were strongly influenced by the changes in the background figures' facial expression. Similarly, Chua, Boland, and Nisbett (2005) presented participants with a set of stimuli consisting of a central object and background scene. Results indicated that Americans looked at central objects sooner and longer and that Asian participants made more eye movements to the background (as well as more total eye movements). In sum, these findings suggest that East Asians' tendency to physically allocate their attention to contextual information is much stronger than that of North Americans.

Identification of the stage where culture can affect vision is beyond the scope of this research. Here, we are content with maintaining that systematic cultural influences, via socialization and probably also environmental affordances, do occur somewhere in the process of vision, and this influence cannot be considered a negligible factor in better understanding the relations between perception and cognition. These findings support the notion that it is differences in attention that underlie other differences in reasoning styles characterized by Nisbett 
and his colleagues as holistic (more typical of East Asians) versus analytic (more typical of Westerners). If attention is distributed quite differently between object and context for Easterners and Westerners, that fact would seem sufficient on the face of it to explain differences in causal attribution: What you see is what you attribute to-and Westerners are relatively more likely to see objects, whereas Easterners are relatively more likely to see contexts.

\section{Notes}

1. East Asian participants refer to those who lived in their countries at least till their high-school or undergraduate-level graduation (undergraduate participants) and who were categorized as international students by university criteria. All the East Asian participants had lived in the United States less than 5 years. American participants were 2 graduate students ( 1 woman and 1 man, age range: $29-31, M=30.00$ ) and 28 undergraduate students (14 females and 14 males, age range: $18-21, M=19.07)$. East Asian participants were 20 graduate students ( 7 women and 13 men, age range: $22-34, M=$ $28.85)$ and 16 undergraduate students (10 women and 6 men, age range: $18-23, M=$ 20.25 ). A 2 (the school status) $\times 2$ (the type of changes) ANOVA was applied to their performance within culture, which indicated that there was no significant interaction between the type of changes and the school status as to their performance, $F<1, n s ; F(1$, $34)=1.07, n s$, respectively. So, we collapsed across the factor of the school status.

2. A 2 (culture: East Asians vs. Americans) $\times 2$ (run out of time: objects vs. contexts) ANOVA indicated that there were no main effects of culture, $F<1$, nor run out of time, $F(1,64)=2.87, n s$. The interaction was not significant, $F<1$.

3. The experimenter first set in advance the central objects in each scene. Next, if participants selected the intended objects, we assigned the value "1." If participants selected unintended objects in the context, we assigned the value " 2. ." Overall, $83.50 \%$ of the participants' data (84\% of Americans' and $83 \%$ of East Asians') were categorized as Value 1 . Then we created a series of contingency tables of culture (Americans vs. Japanese) and selected objects (Value 1 or 2) and carried out chi-square analyses on each scene. The logic was that if there was a significant difference between American and Japanese interpretation of centrality, then the chi-square values should be statistically significant. But, if there are no statistically significant differences in the values, we can conclude that our manipulation was effective, and there are no statistically significant cultural differences in centrality. In a total of 30 chi-square analyses for 30 scenes, no chi-square values were statistically significant, which suggests that there was good agreement between American and East Asian participants.

4. All $p$ values are based on two-tailed tests.

5. To minimize the influence of differences in language, we instructed participants to write their answer as concretely as possible (e.g., "a red car's wheel has been changed). Bilingual coders were trained to become familiar with the types of changes in the scene. Based on their judgment, we accepted relatively vague expressions such as (a part of the front car has been changed) but judged as wrong ambiguous expressions such as "something has been changed." 
6. The pattern of results found in Fig. 5 was found both in the scenes that we regarded as culturally neutral - the airport and construction scenes - and in the culturally specific scenes. Of the possible comparisons between Japanese and Americans, only the difference in report of context differences for the neutral scenes was statistically significant by itself.

7. A three-way interaction was not statistically significant, $F(3,58)=2.25, p>.10$.

\section{Acknowledgments}

This study was supported by National Science Foundation Grants SBR9729103 and BCS0132074., We thank Janxin Leu, Julia S. Carlson, Paul Graham, Nicholas W. Kohn, Keiko Ishii, Carrie Hoi-Suen Lee, Chinae Masuda, Yuri Miyamoto, Mark H. B. Radford, James M. Shields, Margaret Wen-Ching Su, and Mitsuko Yakabi for their assistance.

\section{References}

Bornstein, M. H., Toda, S., Azuma, H., Tamis-LeMonda, C., \& Ogino, M. (1990). Mother and infant activity and interaction in Japan and in the United States II: A comparative microanalysis of naturalistic exchanges focused on the organization of infant attention. International Journal of Behavioral Development, 13, 289-308.

Chalfonte, B. L., \& Johnson, M. K. (1996). Feature memory and binding in young and older adults. Memory and Cognition, 24, 403-416.

Choi, I, Nisbett, R. E., \& Norenzayan, A. (1999). Causal attribution across cultures: Variation and universality. Psychological Bulletin, 125, 47-63.

Chua, H. F., Boland, J., \& Nisbett, R. E. (2005, January). Attention to object vs. background: Eyetracking evidence comparing Chinese and Americans. Poster session presented at the annual meeting of the Society of Personality and Social Psychology, New Orleans, LA.

Chun, M. M. (2000). Contextual cueing of visual attention. Trends in Cognitive Sciences, 4, 170-178.

Fernald, A., \& Morikawa, H. (1993). Common themes and cultural variations in Japanese and American mothers' speech to infants. Child Development, 64, 637-656.

Hedden, T., Ji, L., Jing, Q., Jiao, S., Yao, C., Nisbett, R. E., et al. (2000, April). Culture and age differences in recognition memory for social dimensions. Paper presented at the Cognitive Aging Conference, Atlanta, GA.

Ishii, K., Reyes, J. A., \& Kitayama, S. (2003). Spontaneous attention to word content versus emotional tone: Differences among three cultures. Psychological Science, 14, 39-45.

Ji, L. J., Peng, K., \& Nisbett, R. E. (2000). Culture, control, and perception of relationships in the environment. Journal of Personality and Social Psychology, 78, 943-955.

Kelley, T. A., Chun, M. M., \& Chua, K.-P. (2003). Effects of scene inversion on change detection of targets matched for visual salience. Journal of Vision, 2, 1-5.

Levin, D. T., \& Simons, D. J. (1997). Failure to detect changes to attended objects in motion pictures. Psychonomic Bulletin and Review, 4, 501-506.

Liu, D., \& Nisbett, R. E. (2005). [Contextual effects on the test performance]. Unpublished raw data.

Markus, H., \& Kitayama, S. (1991). Culture and the self: Implications for cognition, emotion, and motivation. Psychological Review, 98, 224-253.

Masuda, T., Ellsworth, P. C., Mesquita, B., Leu, J.-X., Tanida, S., \& van de Veerdonk, E. (2005).

Placing the face in context: Cultural differences in the perception offacial behavior of emotion. Manuscript submitted for publication. 
Masuda, T., \& Kitayama, S. (2004). Perceiver-induced constraint and attitude attribution in Japan and the US: A case for the cultural dependence of the correspondence bias. Journal of Experimental Social Psychology, 40, 409-416.

Masuda, T., \& Nisbett, R. E. (2001). Attending holistically versus analytically: Comparing the context sensitivity of Japanese and Americans. Journal of Personality and Social Psychology, 81, 922-934.

Miyamoto, Y., \& Kitayama, S. (2002). Cultural variation in correspondence bias: The critical role of attitude diagnosticity of socially constrained behavior. Journal of Personality and Social Psychology, 83, 1239-1248.

Miyamoto, Y., Nisbett, R. E., \& Masuda, T. (in press). Differential affordances of Eastern and Western environments. Psychological Science.

Morris, M. W., \& Peng, K. (1994). Culture and cause: American and Chinese attributions for social and physical events. Journal of Personality and Social Psychology, 67, 949-971.

Nisbett, R. E. (2003). The geography of thought: How Asians and Westerners think differently ... and why. New York: Free Press.

Nisbett, R. E., \& Masuda, T. (2003). Culture and point of view. Proceedings of the National Academy of Science, 100, 11163-11175.

Nisbett, R. E., Peng, K., Choi, I., \& Norenzayan, A. (2001). Culture and systems of thought: Holistic vs. analytic cognition. Psychological Review, 108, 291-310.

Norenzayan, A., \& Nisbett, R. E. (2000). Culture and causal cognition. Current Direction in Psychological Science, 9, 132-135.

Peng, K., \& Knowles, E. (2003). Culture, ethnicity and the attribution of physical causality. Personality and Social Psychology Bulletin, 29, 1272-1284.

Rensink, R. A., O'Regan, J. K., \& Clark, J. J. (1997). To see or not to see: The need for attention to perceive changes in scenes. Psychological Science, 8, 368-373.

Ro, T., Russell, C., \& Lavie, N. (2001). Changing faces: A detection advantage in the flicker paradigm. Psychological Science, 12, 94-99.

Scholl, B. J. (2000). Attenuated change blindness for exogenously attended items in a flicker paradigm. Visual Cognition, 7, 377-396.

Simons, D. J. (2000). Current approaches to change blindness. Visual Cognition, 7, 1-15.

Simons, D. J., \& Chabris, C. F. (1999). Gorillas in our midst: Sustained inattentional blindness for dynamic events. Perception, 28, 1059-1074.

Simons, D. J., \& Levin, D. T. (1998). Failure to detect changes to people during a real-world interaction. Psychonomic Bulletin and Review, 5, 644-649.

Tardif, T., Shatz, M., \& Naigles, L. (1997). Caregiver speech and children's use of nouns versus verbs: A comparison of English, Italian, and Mandarin. Journal of Child Language, 24, 535-565. 


\section{Appendix A. Types of Scenes and Changes of the Stimulus Set Used in Experiment 1}

\begin{tabular}{|c|c|c|c|c|}
\hline No. & Scenes & Changes & Change Category & Type \\
\hline 1. & Airport & Front plane's color & Focal object & Property change \\
\hline 2. & Airport & Number of window of the plane & Focal object & Deletion/Addition \\
\hline 3. & Airport & Size of the front plane's engine & Focal object & Form change \\
\hline 4. & Airport & Background control tower's shape & Context & Form change \\
\hline 5. & Airport & Cloud's shape & Context & Form Change \\
\hline 6. & Airport & Helicopter's location & Context & Location \\
\hline 7. & Construction site & Front bulldozer's lights (on/off) & Focal object & Property change \\
\hline 8. & Construction site & Front bulldozer's shape of gear & Focal object & Deletion/Addition \\
\hline 9. & Construction site & Front bulldozer"s line color & Focal object & Form change \\
\hline 10. & Construction site & Number of construction frames & Context & Deletion/Addition \\
\hline 11. & Construction site & Electric lines in background & Context & Deletion/Addition \\
\hline 12. & Construction site & Bulldozer's location & Context & Location \\
\hline 13. & Farm & Pickup truck's door color & Focal object & Property change \\
\hline 14. & Farm & Front tractor's prow & Focal object & Deletion/Addition \\
\hline 15. & Farm & Size of loads on the front tractor & Focal object & Form change \\
\hline 16. & Farm & Height of the background field & Context & Form change \\
\hline 17. & Farm & Airplane's location & Context & Location \\
\hline 18. & Farm & Background barn's location & Context & Location \\
\hline 19. & Harbor & Front steamship's pedal color & Focal object & Property change \\
\hline 20. & Harbor & Logo of the front tag boat & Focal object & Deletion/Addition \\
\hline 21. & Harbor & Front boat's shape of window & Focal object & Form change \\
\hline 22. & Harbor & Location of church steeple & Context & Deletion/Addition \\
\hline 23. & Harbor & Color of water & Context & Form change \\
\hline 24. & Harbor & Ferry's location & Context & Location \\
\hline 25. & Town & Front track's color & Focal Object & Property change \\
\hline 26. & Town & Front bicycle's wheel & Focal object & Deletion/Addition \\
\hline 27. & Town & Front car's wheel shape & Focal object & Form change \\
\hline 28. & Town & Shape of the background building & Context & Deletion/Addition \\
\hline 29. & Town & Shape of the sidewalk & Context & Form change \\
\hline 30. & Town & Track's location & Context & Location \\
\hline
\end{tabular}




\section{Appendix B. Types of Scenes and Changes of the Stimulus Set Used in Experiments 2 and 3}

\begin{tabular}{|c|c|c|c|}
\hline Scenes & Changes in the Scene & Change Category & Subcategory \\
\hline \multirow[t]{11}{*}{ Airport } & Front plane's color & Focal object & Attributes \\
\hline & Helicopter's propeller & Focal object & Attributes \\
\hline & Passenger plane (1)'s logo & Focal object & Attributes \\
\hline & Passenger plane (2)'s number of windows & Focal object & Attributes \\
\hline & Passenger plane (1)'s landing gear & Focal object & Attributes \\
\hline & Passenger plane (1)'s flight course & Context & Movement \\
\hline & Small plane's flight course & Context & Movement \\
\hline & Passenger plane's location (2) & Context & Location \\
\hline & Location of helicopter & Context & Location \\
\hline & Shape of a control tower & Context & Background \\
\hline & Shape of a light pole & Context & Background \\
\hline \multirow[t]{11}{*}{ Construction site } & Backhoe's shape & Focal object & Attributes \\
\hline & Forklift truck's shovel shape & Focal object & Attributes \\
\hline & Forklift truck's window shape & Focal object & Attributes \\
\hline & Bulldozer's color & Focal object & Attributes \\
\hline & Bulldozer's serial number & Focal object & Attributes \\
\hline & Bulldozer's speed & Context & Movement \\
\hline & Forklift truck's speed & Context & Movement \\
\hline & Bulldozer's location & Context & Location \\
\hline & Forklift truck's location & Context & Location \\
\hline & Number of the construction frame & Context & Background \\
\hline & Shape of the ground & Context & Background \\
\hline \multirow{11}{*}{ American harbor } & Tag boat's lifesaver color & Focal object & Attributes \\
\hline & Tag boat's flag color & Focal object & Attributes \\
\hline & Tag boat's number logo & Focal object & Attributes \\
\hline & Tag boat's lifeboat & Focal object & Attributes \\
\hline & Cruiser's lifesaver color & Focal object & Attributes \\
\hline & Tag boat's speed & Context & Movement \\
\hline & Cruiser's speed & Context & Movement \\
\hline & White boat's location & Context & Location \\
\hline & Cruiser's location & Context & Location \\
\hline & Church's location & Context & Background \\
\hline & Shape of a lighthouse & Context & Background \\
\hline \multirow[t]{11}{*}{ American farm } & Air balloon's basket color & Focal object & Attributes \\
\hline & Tractor (1)'s parts & Focal object & Attributes \\
\hline & Tractor (2)'s number of plows & Focal object & Attributes \\
\hline & Tractor (1)’s exhaust pipe & Focal object & Attributes \\
\hline & Truck in the barn & Focal object & Attributes \\
\hline & Tractor (1)'s speed & Context & Movement \\
\hline & Tractor (2)'s speed & Context & Movement \\
\hline & Air balloon's location & Context & Location \\
\hline & Pickup truck's location & Context & Location \\
\hline & Shape of houses & Context & Background \\
\hline & Clouds & Context & Background \\
\hline \multirow[t]{3}{*}{ American town } & Limo's color & Focal object & Attributes \\
\hline & Limo's shape & Focal object & Attributes \\
\hline & Bleu van's window & Focal object & Attributes \\
\hline
\end{tabular}




\begin{tabular}{llll}
\hline Scenes & Changes in the Scene & Change Category & Subcategory \\
\hline & Red car's shape & Focal object & Attributes \\
Shape of a red car's wheel & Focal object & Attributes \\
Blue car's speed & Context & Movement \\
Silver car's speed & Context & Movement \\
Red car's location & Context & Location \\
Limo's location & Context & Location \\
Shape of the middle building & Context & Background \\
Shape of the left building & Context & Background \\
Tractor (2) driver's clothes & Focal object & Attributes \\
Tractor (1) driver's helmet & Focal object & Attributes \\
Number of loads on the tractor (2) & Focal object & Attributes \\
Tractor (1)'s color & Focal object & Attributes \\
Red tractor's parts & Focal object & Attributes \\
Tractor (1)'s speed & Context & Movement \\
Tractor (2)'s speed & Context & Movement \\
Red tractor's location & Context & Location \\
Tractor (2)'s location & Context & Location \\
& Shape of houses & Context & Background \\
& Clouds & Context & Background \\
& Postal car's symbol & Focal object & Attributes \\
& Cleaning car's back parts & Focal object & Attributes \\
& Police car's shape & Focal object & Attributes \\
Police car's logo & Focal object & Attributes \\
& Police car's parts & Focal object & Attributes \\
Postal car's location & Context & Movement \\
Cleaning car's location & Context & Movement \\
Green plane's location & Context & Location \\
Postal car's original location & Context & Location \\
Shape of background buildings & Context & Background \\
Electric lines & Context & Background \\
\hline & &
\end{tabular}

